\title{
miR-27b shapes the presynaptic transcriptome and influences neurotransmission by silencing the polycomb group protein Bmi1
}

\author{
Vivian Y. Poon ${ }^{1+}$, Minxia Gu${ }^{1+}$, Fang $\mathrm{Ji}^{1}$, Antonius M. VanDongen ${ }^{1}$ and Marc Fivaz ${ }^{1,2^{*}}$ (D)
}

\begin{abstract}
Background: MicroRNAs (miRNAs) are short non-coding RNAs that are emerging as important post-transcriptional regulators of neuronal and synaptic development. The precise impact of miRNAs on presynaptic function and neurotransmission remains, however, poorly understood.

Results: Here, we identify miR-27b—an abundant neuronal miRNA implicated in neurological disorders—as a global regulator of the presynaptic transcriptome. miR-27b influences the expression of three quarters of genes associated with presynaptic function in cortical neurons. Contrary to expectation, a large majority of these genes are up-regulated by miR-27b. This stimulatory effect is mediated by miR-27b-directed silencing of several transcriptional repressors that cooperate to suppress the presynaptic transcriptome. The strongest repressive activity appears to be mediated by Bmi1, a component of the polycomb repressive complex implicated in self-renewal of neural stem cells. miR-27b knockdown leads to reduced synaptogenesis and to a marked decrease in neural network activity, which is fully restored by RNAi-mediated silencing of Bmi1.

Conclusions: We conclude that silencing of Bmi1 by miR-27b relieves repression of the presynaptic transcriptome and supports neurotransmission in cortical networks. These results expand the repressive activity of Bmi1 to genes involved in synaptic function and identify a unique post-transcriptional circuitry that stimulates expression of synaptic genes and promotes synapse differentiation.
\end{abstract}

Keywords: miRNAs, Synapse, Transcriptome, Polycomb Group Protein, Neurotransmission, Epigenetics

\section{Background}

miRNAs are small noncoding RNAs that regulate gene expression by base pairing to mRNAs. Targeting occurs by complementarity between the seed region ( $6-8 \mathrm{bp})$ of the miRNA and the mRNA 3' UTR [1], and results in transcript silencing by translational repression and mRNA destabilization [2-4]. This minimal degree of base pairing enables a single miRNA to target multiple (tens to hundreds) transcripts $[5,6]$ thereby complicating the identification of relevant miRNA targets for a given

\footnotetext{
* Correspondence: marc.fivaz@duke-nus.edu.sg

${ }^{\dagger}$ Equal contributors

'Program in Neuroscience and Behavioral Disorders, Duke-NUS Medical School, Singapore, Singapore

${ }^{2}$ Department of Physiology, Yong Loo Lin School of Medicine, National University of Singapore, Singapore, Singapore
}

biological outcome. On the other hand, a single gene is typically under the control of several different miRNAs. Consequently, miRNA networks appear highly distributed, with each individual miRNA having a widespread but modest effect on gene expression.

miRNAs are abundant in the brain and have been implicated in various aspects of CNS development, from renewal of neural stem cells, to differentiation, neurite projection, dendrite maturation and synaptic plasticity [7]. The impact of miRNAs on neurodevelopment in both vertebrates and invertebrates is now supported by a large body of work (reviewed in [7]), and provides a plausible explanation for the increasing number of miRNAs associated with neurodevelopmental disorders [8]. Recent studies also point to a role of miRNAs at the synapse, 
particularly on the post-synaptic side. Specific miRNAs control dendrite complexity [9], dendritic excitability $[10,11]$ and spine shape [12-14], while others target glutamate receptors $[15,16]$ and post-synaptic scaffolds [17], or regulate Long-Term Potentiation (LTP) and Depression (LTD) [18, 19], persistent forms of synaptic plasticity that are thought to rely predominantly on post-synaptic mechanisms.

Comparatively less is known about the effect of miRNAs on presynaptic functions and neurotransmitter release. This is somewhat surprising, given that proper dosage of neurotransmitter release is of central importance for neural circuit performance and is fine-tuned by complex gene regulatory networks [20]. In support, however, of a role of miRNAs in this process, a recent bioinformatics study shows that presynaptic transcripts have an unusually long 3'UTR with an increased number of predicted miRNA target sites [21]. In addition, miR-1000 and the miR-310 cluster target presynaptic components and regulate neurotransmitter release in flies [22, 23].

We have previously identified miR-27b in a targeted screen for miRNAs that regulate presynaptic assembly in rodent cortical neurons [24]. miR-27b is highly-expressed in the brain [5] and has been associated with bipolar disorders and schizophrenia [25]. Here, we explore the impact of miR-27b on $\sim 200$ transcripts that make up the presynaptic compartment in excitatory neurons. We find, unexpectedly, that miR-27b up-regulates the expression of more than half of these presynaptic transcripts. This enhancing effect is due to miR-27b-dependent down-regulation of three main transcriptional repressors-Bmil, Sox11 and Zfp90-that operate in concert to silence the presynaptic transcriptome. Our results further indicate that miR-27b-dependent silencing of Bmil shapes neural activity in cortical networks and suggest that posttranscriptional repression of Bmil is required for the developmental transition of a neural stem cell into a mature, synaptically-competent neuron.

\section{Results}

miR-27b boosts expression of presynaptic genes

To explore the global impact of miR-27b on genes associated with presynaptic function, we first compiled a list of 195 "presynaptic" genes, based on previous proteomics and functional studies (see Methods). These genes encode for the vast majority of proteins that make up the presynaptic compartment, and include synaptic vesicle (SV) proteins, active zone proteins, channels, adhesion molecules, trafficking and signaling proteins (Fig. 1a; Additional file 1: Table S1). We have also included in that list a handful of neuronally expressed and validated miR-27b and Dicer1 targets, many of which regulate gene expression (Additional file 1: Table S1). To knock down miR-27b in cortical neurons, we targeted miR-27b-3p-the guide strand of the miR-27b duplex and one of the most abundant miRNAs loaded onto the RNA-induced silencing complex (RISC) in the brain [6]-using a shRNA (miRZip-27b) cloned into a GFP-expressing lentiviral vector. Transduction of cortical neurons with miRZip-27b led to a specific and substantial reduction of miR-27b-3p levels (referred to hereafter as miR-27b) whereas a scrambled shRNA sequence (miRZip-Scr) had no effect (Fig. 1b). Because mammalian miRNAs silence gene expression predominantly by promoting message destruction [2], we profiled the mRNAs of these $\sim 200$ genes both in miR-27b knockdown (KD) and control (CT) neurons. As miRNAs typically have mild effects on the expression of most genes, we used a profiling approach (nCounter, Nanostring) that offers high levels of precision and sensitivity [26] and which is based on color-coded, gene-specific barcodes and multiplex single-molecule imaging of individual transcripts.

Analysis of differential gene expression showed that miR-27b significantly regulates 147 genes, which amounts to $76 \%$ of the presynaptic transcriptome after the exclusion of 14 genes with low counts (Fig. 1c-e; Additional file 2: Table S2A). To our surprise, $70 \%$ of the affected genes were down-regulated implying a positive influence of miR-27b on gene expression. To determine whether this type of regulation is unique to miR-27b, we profiled these transcripts in cortical neurons derived from a mouse homozygous for the floxed Dicer1 allele (Dicer $\left.{ }^{f l / f l}\right)$ and transduced with the Cre recombinase (or a control vector). We reasoned that if most miRNAs repress presynaptic genes, we should see an overall increase in gene expression in cells devoid of miRNAs. Transduction of Cre in Dicer $\mathrm{f}^{f l / f l}$ neurons led to marked reduction of miR-27b and miR-181a, consistent with efficient Cremediated excision of the Dicer1 gene (Fig. 1f). Among the 140 genes that are differentially regulated in Dicer 1 cKO neurons, 128 (91\%) were up-regulated, in agreement with a global negative impact of miRNAs on gene expression (Fig. 1g; Additional file 2: Table S2B). Thus, in contrast to the bulk of miRNAs, miR-27b exerts a positive influence on the presynaptic transcriptome.

\section{The transcriptional regulators Bmi1, Sox11 and Zfp90 are direct targets of miR-27b}

We hypothesized that the stimulatory effect of miR-27b on gene expression is indirect and is mediated by miR27b-dependent silencing of transcriptional repressors or mRNA-destabilizing genes. To systematically search for putative miR-27b targets, we first analyzed the impact of miR-27b on gene expression at the genome-wide level using a microarray approach. Differential gene expression analysis revealed a total of 860 and 851 genes that were up- and down-regulated, respectively (fold-change $>1.5$, false discovery rate $<0.05$, Fig. 2a; Additional file 3 : Table S3). Comparative analysis of the presynaptic transcriptome in the nCounter and microarray datasets 


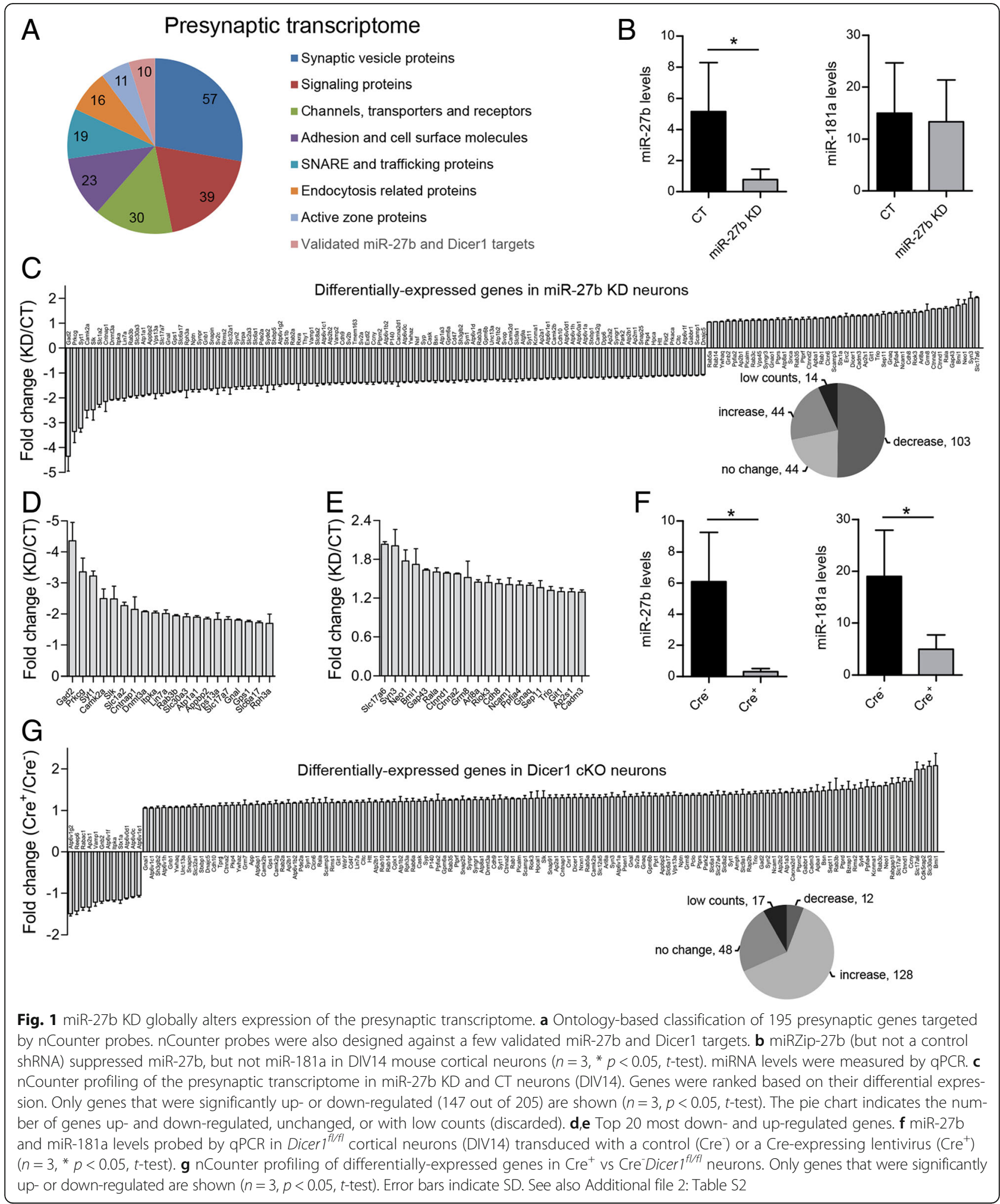

reveals a $50 \%$ overlap among the top 40 most differentially-expressed genes (Additional file 4: Table S4). Preferential down-regulation of presynaptic genes in miR27b KD neurons is also observed in the microarray dataset
(Additional file 5: Figure S1), but disappears at the genome-wide level (Fig. 2a; Additional file 3: Table S3), suggesting the stimulating activity of miR-27b only applies to a targeted subset of genes. 


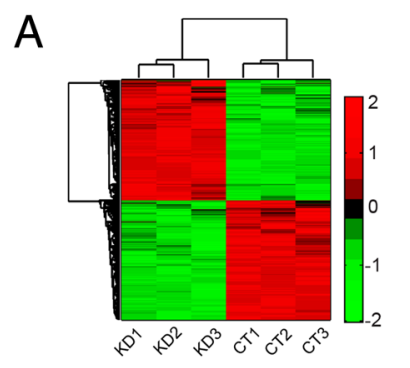

B

C

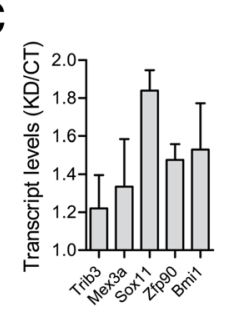

D

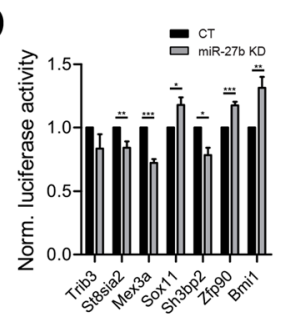

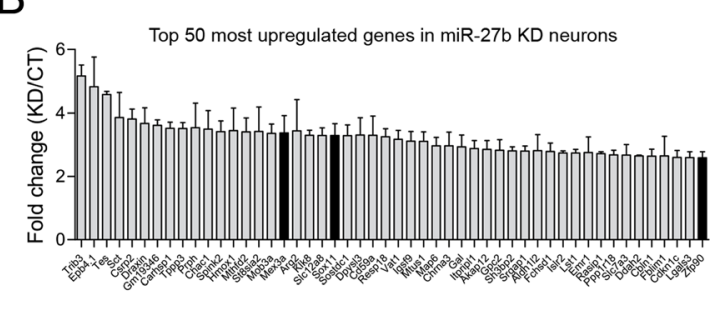

E

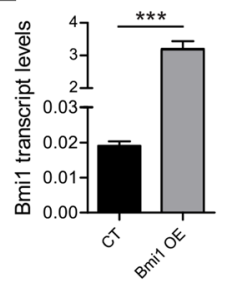

F

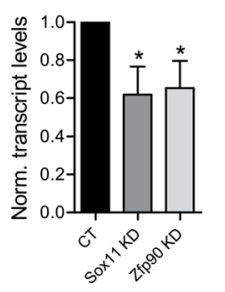

G

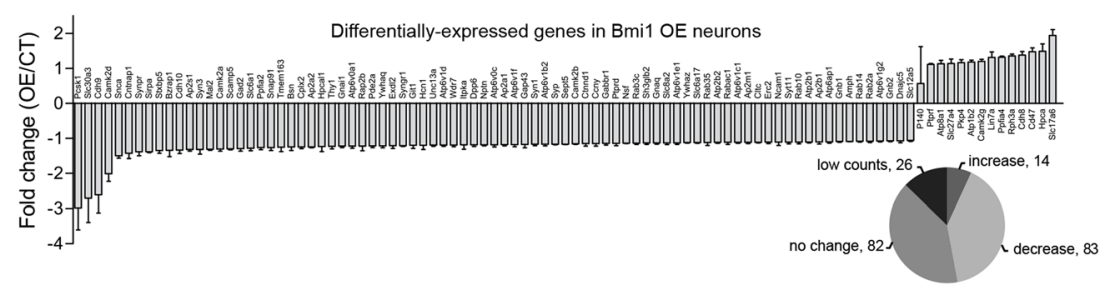

$\mathrm{H}$

I
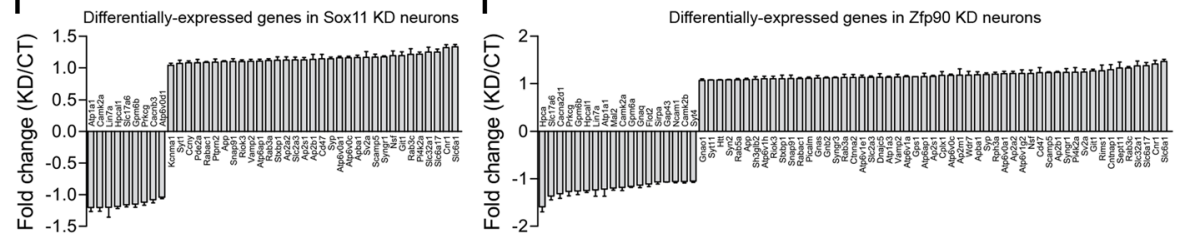

J

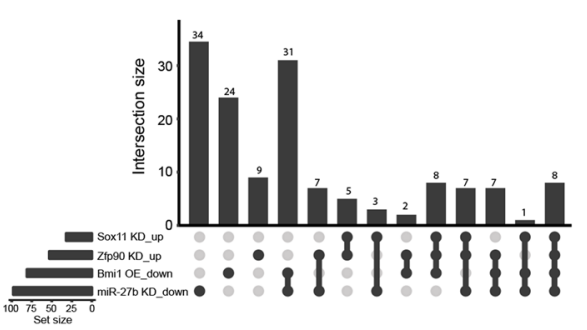

K

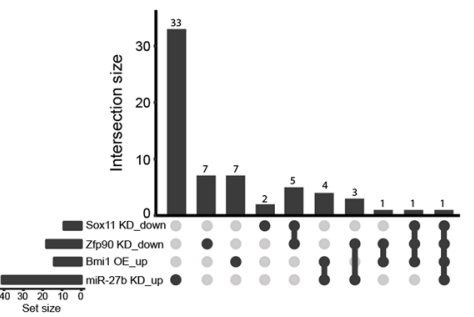

Fig. 2 Sox11, Zfp90 and Bmi1 are miR-27b targets. a,b Genome-wide transcriptome analysis of miR-27b KD and CT mouse cortical neurons (DIV14). a Hierarchical clustering of differentially-expressed genes with fold-change $\geq 1.5$ (FDR $<0.05)$. Columns represent individual experiments (KD, miR-27b knockdown; CT, miR-27b control). Rows correspond to individual genes. (See also Additional file 3: Table S3). b Top 50 most up-regulated genes in miR-27b KD neurons $(n=3, F D R<0.006)$. Dark grey bars refer to candidate repressor genes. $\mathbf{c}$ qPCR analysis of Trib3 (the most-upregulated gene in the microarray data) and candidate repressors in miR-27b KD vs CT neurons $(n=4)$. Results are expressed as the KD/CT ratio. $\mathbf{d}$ Luciferase reporters containing the $3^{\prime} U T R$ of candidate repressors (and a few other genes listed in (b)) were electroporated in neurons later transduced with miRZip-27b and miRZip-Scr lentiviruses. Luciferase activities were normalized to CT values (set to 1). (Trib3 and St8sia2, $n=5 ;$ Mex3a, $n=6$; Sox11 and Zfp90, $n=7$; Sh3bp2, $n=4 ;$ Bmi1, $n=9 .{ }^{*} p<0.05,{ }^{* *} p<0.01,{ }^{* *} p<0.001, t$-test). e qPCR analysis of Bmi1 expression in neurons (DIV14) transduced with a control vector or a Bmi1-expressing construct ( $n=4{ }^{* * *} p<0.001$, $t$-test). $\mathbf{f}$ RNAi KD of Sox11 and Zfp90 in cortical neurons assessed by $\mathrm{qPCR}\left(n=3,{ }^{*} p<0.05\right.$, t-test). $\mathbf{g}$ nCounter profiling of the presynaptic transcriptome in CT and Bmi1 OE cortical neurons $(n=4, p<0.05$, $t$-test). 97 genes are differentially regulated. $\mathbf{h}, \mathbf{i} \mathrm{nCounter}$ analysis of the presynaptic transcriptome in Sox 11-and Zfp90-silenced cortical neurons $(n=3, p<0.05) .42$ and 71 genes are differentially-expressed respectively. Error bars are SD except for C-E where they represent SEM. $\mathbf{j}, \mathbf{k}$ Combinatorial intersection analysis of differentially-expressed genes in miR-27b KD, Bmi1 OE, Sox11- and Zfp90 KD neurons. $\mathbf{j}$ Intersections among genes repressed by Bmi1, Sox 11 and Zfp90 and activated by miR-27b. $\mathbf{k}$ Intersections among genes repressed by miR-27b and activated by Bmi1, Sox11 and Zfp90 
Next, we searched for candidate genes based on the extent of their up-regulation in miR-27b KD neurons, the presence of predicted miR-27b-binding sites in their 3'-UTR, and their reported function as transcriptional or post-transcriptional regulators. Three genes, Mex3a, Sox11 and $Z f p 90$ were selected based on these criteria (Fig. 2b). We added to that list Bmi1, the 4th most upregulated gene in the nCounter dataset (Fig. 1e) and a validated miR-27b target [27]. We confirmed by qPCR that all four genes are upregulated in miR-27b KD neurons (Fig. 2c), and tested the ability of miR-27b to downregulate luciferase reporters containing the 3'-UTRs of these putative repressors. Reporters with the 3'-UTRs of Bmil, Sox11 and Zfp 90 , are up-regulated in miR-27b KD neurons (Fig. 2d), indicating that these three genes are likely targets of miR-27b.

\section{miR-27b turns on the presynaptic transcriptome by silencing Bmi1, Sox11 and Zfp90}

Bmil is a polycomb group protein that functions as an epigenetic repressor [28] and maintains self-renewal of neural stem cells [29]. Sox11 is a member of the SoxC family of transcription factors that regulates neurogenesis in both the central and peripheral nervous system [30-32]. De novo Sox11 mutations cause Coffin-Siris syndrome, a congenital disorder characterized by microcephaly and intellectual disability [33]. Less is known about the zinc-finger protein Zfp90. This transcription factor regulates the transcriptional repressor REST [34] and is located in a 16q22.1 microdeletion in a family with mental retardation [35].

To determine which, if any, of these three transcriptional regulators mediate miR-27b's effect on the presynaptic transcriptome, we profiled presynaptic transcripts in neurons with altered levels of Bmi1, Sox11 and Zfp90. Each of these three transcriptional regulators functioned, globally, as a repressor (Fig. 2g-i). Overexpression of Bmil (Fig. 2e) resulted in repression of 81 genes, 47 of which (58\%) overlapped with miR-27b up-regulated genes (Fig. 2j). Only a dozen genes were upregulated by Bmil, with little intersection (5 out of 41, 12.2\%) with miR-27b repressed genes (Fig. 2k). The striking similarity between Bmi1-repressed and miR-27b up-regulated genes suggests that Bmil is a major functional target of miR-27b.

Because we were unable to overexpress Sox11 and Zfp90 at sufficient levels (not shown), we silenced them, instead, by lentiviral delivery of shRNAs. This approach resulted in a moderate $(\sim 40 \%)$ reduction of Sox11 and Zfp90 transcripts (Fig. 2f). Fewer presynaptic genes are significantly regulated by Sox11 or Zfp90 (Fig. 2h,i), which could reflect incomplete knockdown of these transcriptional regulators. Among the genes repressed by Sox 11 and Zfp90 (i.e. up-regulated in Sox11- and Zfp90-silenced neurons), 19 (59\%) and 29 (55 \%) intersected, respectively, with miR-27b upregulated genes (Fig. 2j). More than
$47 \%$ of Sox11- and Zfp90-repressed genes were corepressed by Bmil, while Sox 11 and Zfp90 shared $87 \%$ of their down-regulated target genes (Fig. 2j). Only a handful of genes were up-regulated by Sox11 and Zfp90 (Fig. 2h,i) with little overlap with miR-27b repressed, or Bmi1 stimulated genes (Fig. 2k). Thus, there is significant overlap in genes repressed by Bmil, Sox11 and Zfp90 and silencing of these three transcriptional regulators by miR-27b likely has an additive effect on the expression of presynaptic genes. These data led us to infer that miR-27b shapes the presynaptic transcriptome by silencing at least three transcriptional repressors with intersecting target genes.

Gene set enrichment analysis predicts roles of miR-27b in synaptic transmission and repression of a proliferative state We were intrigued by the fact that two out of three repressors of the presynaptic transcriptome-Bmil and Sox11-are central regulators of neural stem cell renewal and neurogenesis. This led us to speculate that miR-27b functions as a genetic switch by suppressing proliferation and promoting synapse maturation.

To test this hypothesis further, we performed a gene set enrichment analysis (GSEA) [36, 37] on the microarray data described earlier, to find out whether gene sets related to synaptic transmission and cell proliferation are systematically altered in miR-27b KD neurons. We first ranked all genes according to the extent of their differential expression in miR-27b KD and CT cells. We then computed normalized enrichment scores (NES) for a collection of 1330 curated gene sets representing canonical biological pathways, and identified gene sets that are overrepresented at both extremes of the ranked list. Among the top 60 gene sets associated with down-regulated genes in miR-27b KD neurons, 13 of them ( $22 \%)$ were related to neuronal excitability, synaptic transmission, neurotransmitter release and synaptic plasticity (Fig. 3a, b; Additional file 6: Table S5). At the other extreme, up-regulated genes belonged to gene sets (12 out of the top 60, $20 \%$ ) associated with DNA synthesis and the cell cycle (Fig. 3c, d; Additional file 7: Table S6). To functionally visualize these enrichment results, we displayed them as a network where pathways are nodes and edges represent pathway crosstalk [38]. This analysis revealed two main functional gene modules related to synaptic transmission and DNA synthesis (Fig. 3e and Additional file 8: Figure S2). Together, these data show that miR-27b reciprocally regulates gene networks associated with neurotransmission and cell proliferation.

To identify miR-27b-regulated genes potentially involved in neurotransmission, we extracted core members of the transmission-across-chemical-synapse gene set (Fig. 3a) that contribute to the enrichment score. Hierarchical clustering of these genes shows, as expected, that they are significantly down-regulated in miR-27b KD neurons (Fig. 3f). Many of these genes have clear 


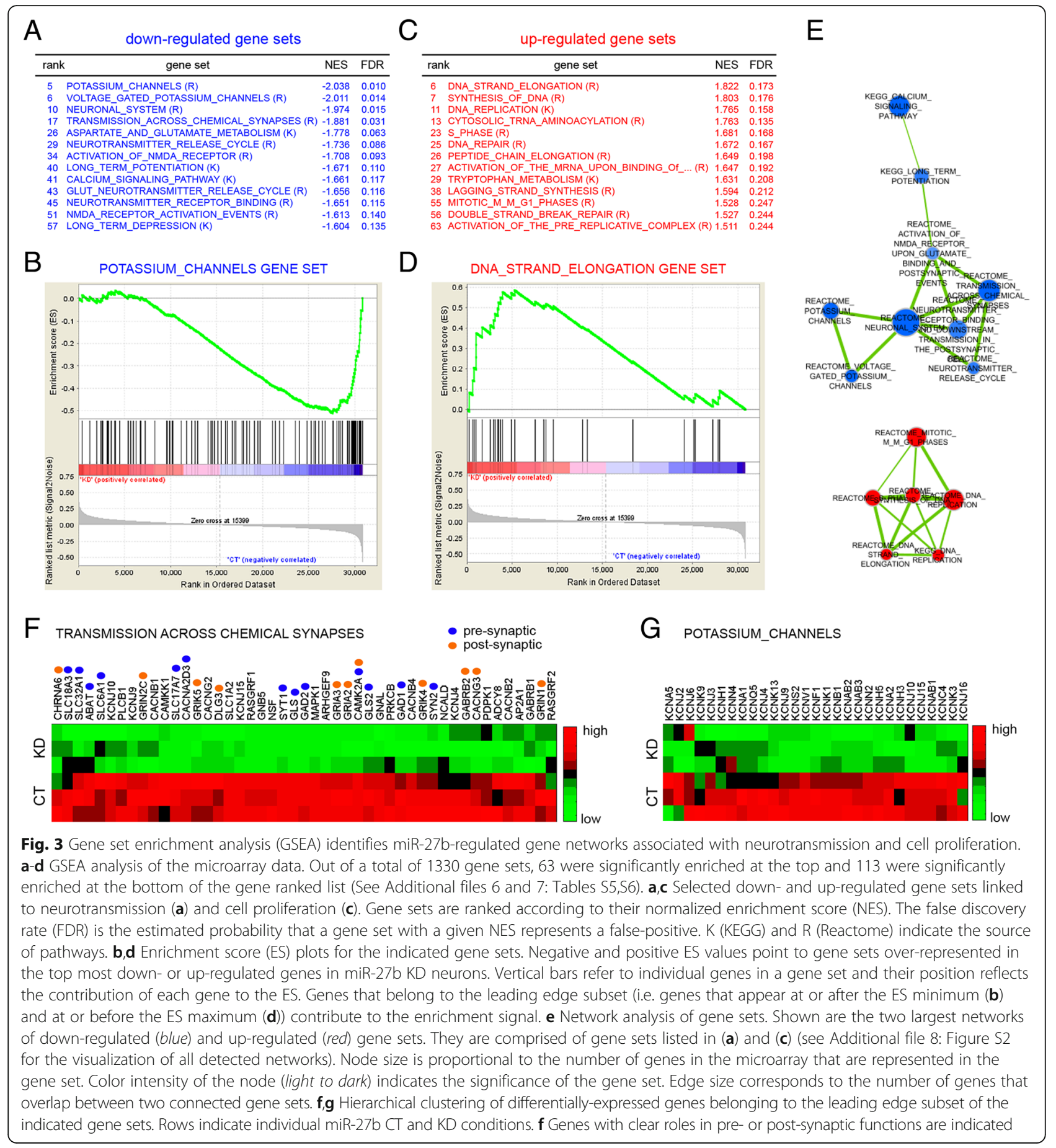

presynaptic functions and five of them, Gad2 (GAD65, Glutamate Decarboxylase 2), Syt1 (Synaptotagmin 1), Slc17a7 (VGLUT1, Vesicular Glutamate Transporter), Slc32a1 (VGAT, Vesicular GABA Transporter) and Syn2 (Synapsin II) belong to the top 30 most down-regulated genes in the nCounter dataset (Fig. 1c; Additional file 2: Table S2). Of interest, these genes include regulators of both glutamate and GABA metabolism and transport, pointing to a role of miR-27b in both excitatory and inhibitory neurotransmission. A similar number of genes encode for postsynaptic proteins (Fig. 3f), including AMPA-receptor (Gria2 and Gria3), NMDA-receptor (Grin1 and Grin2c) and kainate-receptor (Grik4) subunits, suggesting both pre- and post-synaptic functions for miR27b. Moreover, the positive impact of miR-27b on the expression of 29 potassium channels (Fig. 3g), most of 
which are voltage-gated, hints at a broader role of miR-27b in neuronal excitability.

\section{miR-27b stimulates neural network activity by repressing Bmi1}

These GSEA results prompted us to examine the effect of miR-27b on synaptic transmission. We first measured miniature excitatory post-synaptic currents (mEPSCs) in miR-27b KD and CT neurons by whole-cell patch clamp recordings. mEPSCs are driven by spontaneous release of glutamate from excitatory inputs, in the absence of action potential (AP) firing. The frequency of mEPSCs, but not their amplitude, was markedly decreased in miR-27b KD neurons (Fig. 4a-c). While mEPSC amplitude is governed by the number and/or activity of AMPARs on the post-synaptic membrane, mEPSC frequency depends on the probability of neurotransmitter release and the number of excitatory inputs on the recorded neuron. To find out whether miR-27b regulates the formation of excitatory synapses, we immunostained vGLUT1, a marker of excitatory synaptic vesicles, in miR-27b KD and CT neurons. Because miR-27b also boosts expression of genes linked to GABA neurotransmission (Figs. 1d and 3f) we also labeled the SV-resident GABA transporter VGAT in these cells. As anticipated, both SV markers label distinct populations of boutons and excitatory synapses far exceed inhibitory synapses in these neuron cultures (Fig. 4d). A quantitative analysis of these puncta shows a marked reduction (about $50 \%$ ) in both glutamatergic and GABAergic terminals (Fig. 4d-f). Surprisingly, however, the average intensity of VGLUT1 and VGAT puncta is unaffected by miR-27b knockdown, despite significant downregulation of VGLUT and VGAT transcripts in miR27b KD neurons (Fig. 3f; Additional file 2: Table S2A), suggesting that miR-27b preferentially regulates the number of synapses rather than the abundance of these SV markers at synaptic terminals. The stimulatory effect of miR-27b on synaptogenesis was further confirmed using the active zone scaffold bassoon as a presynaptic marker (Additional file 9: Figure S3). Therefore, reduced number of excitatory synaptic inputs likely contributes to decrease in mEPSC frequency in miR-27b KD neurons.

To evaluate the impact of miR-27b on neuronal excitability and AP firing, we measured spontaneous network activity in miR-27b KD and CT cortical neuron cultures using multi-electrode arrays (MEAs). After 2 weeks in vitro, these neuronal networks exhibit spontaneous activity consisting of single spikes and bursts that can be recorded in real-time and non-invasively on an MEA chip (Fig. 4g). Control cultures display an average of $61.3 \pm$ 7.6 SEM bursts in $5 \mathrm{~min}$ (Fig. 4h). Average burst frequency was markedly reduced, however, in miR-27b KD neurons $(26.5 \pm 5.5 \mathrm{SEM})$. To determine whether miR$27 \mathrm{~b}$ boosts network activity by repressing Bmi1, we co- silenced miR-27b and Bmi1 by transducing neurons with both miRZip-27b and a Bmil-targeting shRNA, in an attempt to restore network activity. We verified first that Bmi1 levels are efficiently brought down in miR-27b KD neurons by knockdown of Bmi1 (Fig. 4i). Neuron cultures co-expressing miRZip-27b and a Bmil shRNA fire bursts at a frequency similar to control cells $(57.1 \pm 4.8$ SEM), providing evidence for a role of Bmil in miR-27bdependent regulation of network activity (Fig. 4h). Collectively, these data show that miR-27b boosts neurotransmission by acting, at least in part, on the presynaptic compartment and suggest that de-repression of the presynaptic transcriptome by miR-27b silencing of Bmil is sufficient to support efficient neurotransmission in neural networks.

\section{Discussion}

Previous analyses of miRNA-mRNA networks have suggested that miRNAs function primarily as rheostats-they buffer gene expression but rarely act as master regulators $[39,40]$. miR-27b appears to be an exception to this rule. By silencing three transcriptional repressors that target overlapping sets of genes, miR-27b has a profound impact on the presynaptic transcriptome. More than half (54\%) of all genes that make up the presynapse are positively influenced by miR-27b. The stimulatory effect of miR-27b on presynaptic genes is greater than its silencing activity, both in terms of magnitude (i.e fold-change in gene expression) and number of genes affected. This suggests the presence of an underlying amplification mechanism, which, we propose, is mediated by the cooperative repressive activity of three miR-27b targets. The high fraction of genes co-regulated by miR-27b and Bmi1 (58 \%) suggest that silencing of this epigenetic repressor significantly contributes to miR-27b-dependent up-regulation of presynaptic genes. Rescue of network activity in miR-27b-silenced cortical neurons by RNAi KD of Bmi1 supports a functional role of this epigenetic repressor downstream of miR-27b. Because most genes repressed by Sox11 and Zfp90 overlap with those targeted by Bmi1, there remains about $40 \%$ of miR-27b up-regulated genes that could potentially be under the control of other repressors.

Bmil is a core component of the Polycomb Repressive Complex 1 (PRC1), which, together with PRC2, is widely deployed in eukaryotic cells to implement gene silencing [41]. Bmi1 drives self-renewal of neuronal stem cells in the forebrain and cerebellum by suppression of cell cycle inhibitors [42, 43]. Our data now show that Bmil also represses a significant fraction of genes associated with presynaptic functions, suggesting a novel mode of action of this epigenetic regulator in maintaining an undifferentiated state. By promoting cell proliferation and repressing genes implicated in neurotransmission, Bmi1 puts a two-tier break on cell differentiation. We propose that 


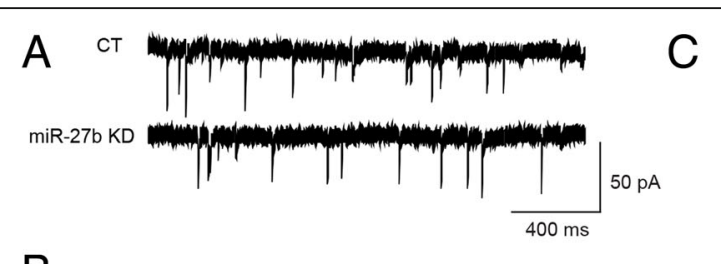

B
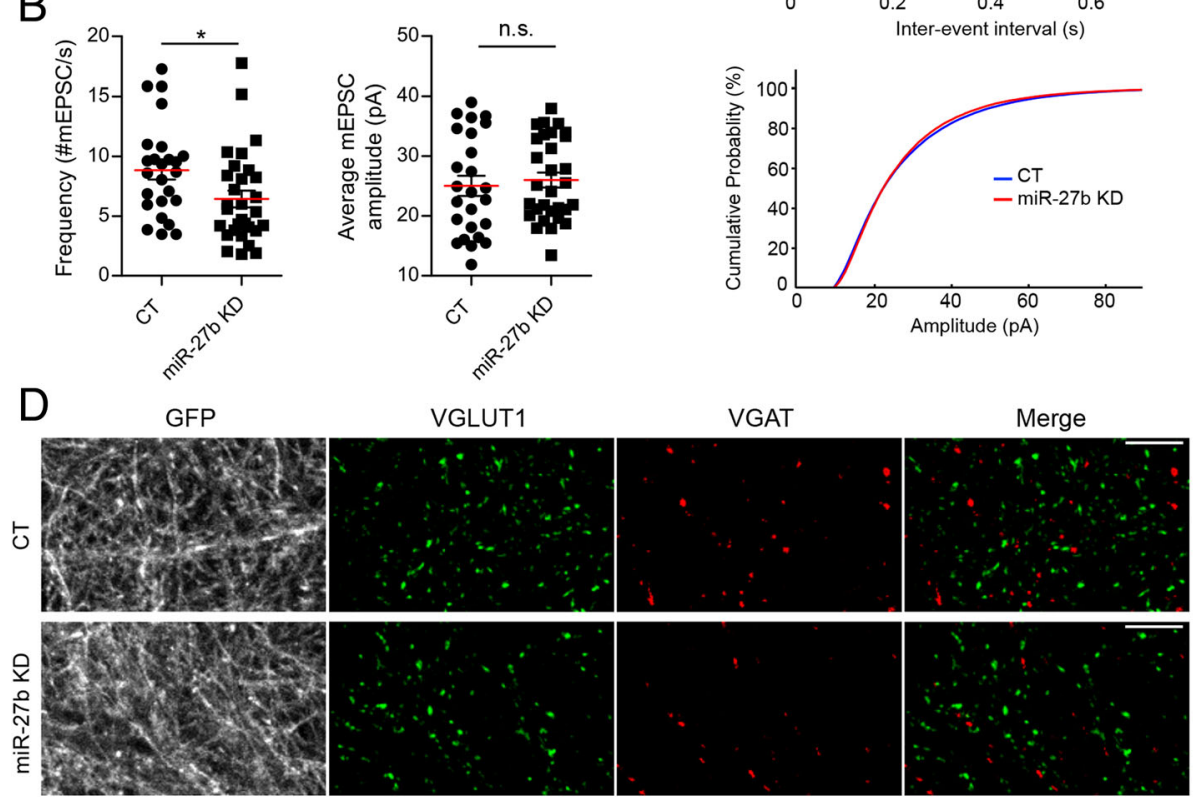

E

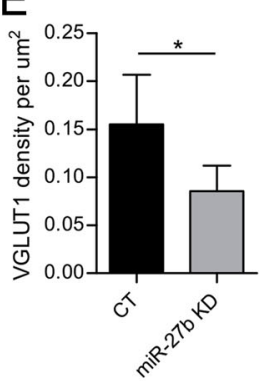

G

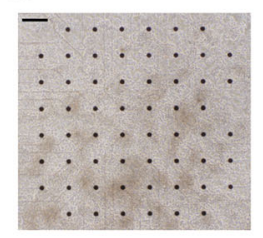

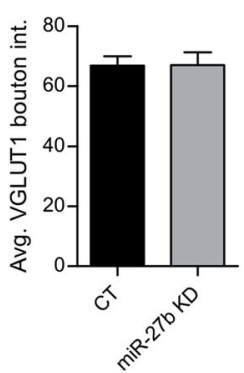

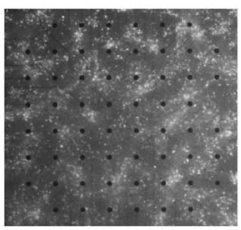

F
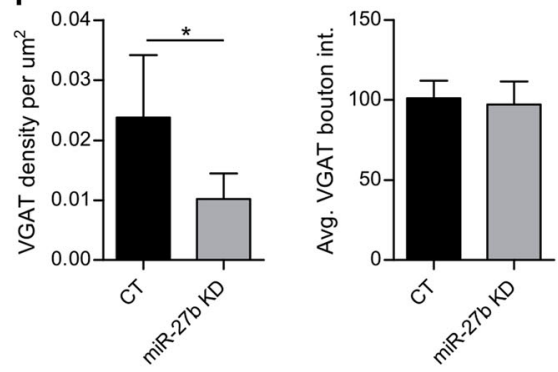

$\mathrm{H}$

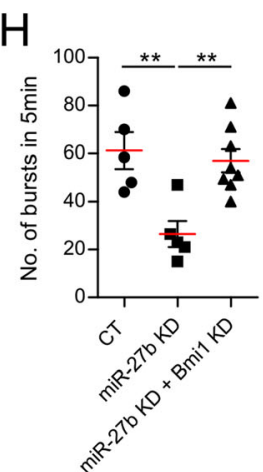

$-\mathrm{CT}$

${ }_{-\mathrm{miR}-27 \mathrm{bD}}$
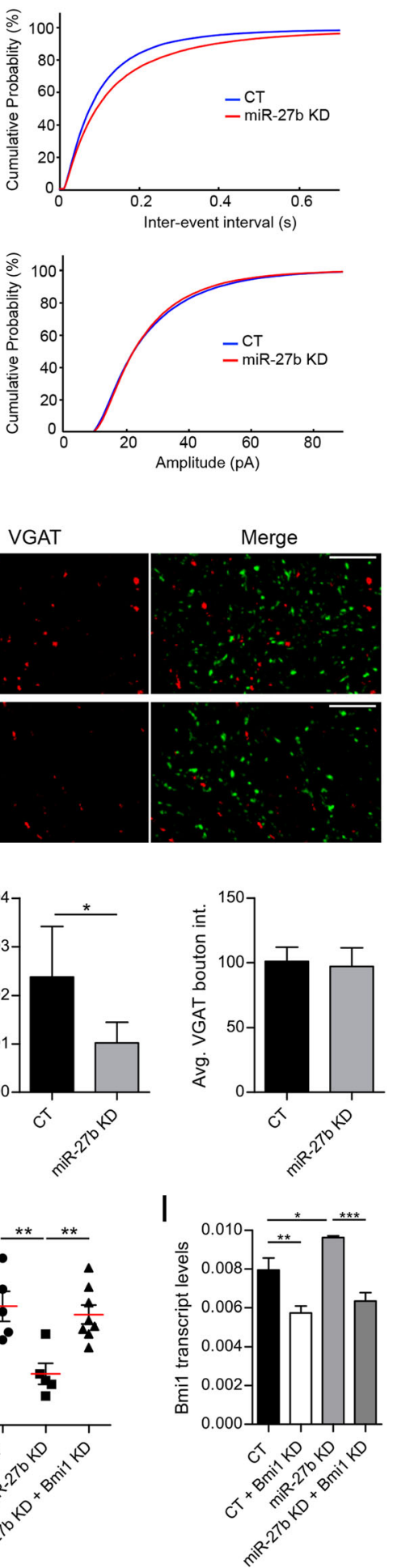

Fig. 4 (See legend on next page.) 
(See figure on previous page.)

Fig. 4 miR-27b enhances neurotransmission. a-c Whole-cell patch clamp recordings of mEPSCs in miR-27b KD and CT hippocampal neurons (DIV14). a Representative examples of individual mEPSC traces for both conditions. $\mathbf{b}$ Quantification of mEPSC frequency and amplitude in miR-27b CT $(n=25)$ and $\mathrm{KD}(n=30) .{ }^{*} p<0.05, t$-test. Red bars indicate the Mean and error bars represent SEM. Each symbol corresponds to data from a single neuron. c Cumulative distributions of mEPSC frequency and amplitude. $\mathbf{d}-\mathbf{f}$ Immunostaining of VGLUT1 and VGAT in miR-27b KD and CT neurons. Scale bar: 10um. e,f Quantification of puncta density and intensity for (e) VGLUT1 (16 fields) and (f) VGAT (16 fields) from 3 experiments. ${ }^{*} p<0.05, t$-test. g-i MEA recordings of miR-27b CT and KD cortical neurons (DIV15), or miR-27b KD neurons transduced with a Bmi1-targeting shRNA. g Bright-field (left) and mCherry fluorescence (right) images of a neuron network grown on an MEA chip. mCherry reports expression of miRZip-27b. Scale bar: 200um. $\mathbf{h}$ Quantification of burst frequency for the indicated conditions. Each symbol represents bursting activity of a single MEA culture dish. MEA dishes originate from three independent experiments. Red bars indicate the Mean (** $p<0.01$, one-way ANOVA). $\mathbf{i}$ qPCR detection of Bmi1 transcripts in cortical neurons expressing miRZip-Scr, Bmi1-shRNA, miRZip-27b and miRZip-27b +Bmi1-shRNA $\left(n=7,{ }^{*} p<0.05\right.$, ** $p<0.01$, *** $p<0.001$, one-way ANOVA). Error bars represent SEM

miR-27b-directed silencing of Bmi1 (and other repressors such as Sox11 and Zfp90) relieves this break and drives (pre)synaptic maturation of excitatory neurons. This model is supported by our GSEA results, which identify neurotransmission and cell proliferation as the largest and most significant gene networks reciprocally regulated by miR-27b. In this context, it is worth mentioning the opposite effect of miR-27b on VGLUT1 (Slc17a7) and VGLUT2 (Slc17a6) expression-VGLUT1 is down-regulated 1.8-fold, while VGLUT2 is the most up-regulated presynaptic gene in miR-27b KD neurons (nCounter dataset). A clear reciprocal expression of these two vesicular glutamate transporters is also observed in the microarray data set. Of note, VGLUT1 and VGLUT2 mark distinct, largely non-overlapping populations of glutamatergic neurons in the CNS-VGLUT1 is expressed in the neocortex (layers I-III), hippocampus and amygdala, while VGLUT2 is found in layer IV of the cerebral cortex, dentate gyrus, thalamus and hypothalamus [44]. This suggests the possibility that miR-27b promotes differentiation of specific, VGLUT1-positive, lineages of excitatory neurons. Strikingly, VGLUT2 is also the most up-regulated gene in Bmil-overexpressing cells, indicating that its repression in cortical neurons may also occur through miR-27b-directed silencing of Bmi1. The stimulatory effect of miR-27b on synapse development is not, however, restricted to excitatory neurons. Knockdown of miR-27b also results in downregulation of GABA specific genes and in reduced density of inhibitory synaptic terminals, suggesting wider functions of miR-27b in excitatory and inhibitory balance.

Given the broad impact of miR-27b on the presynaptic transcriptome, and its influence on post-synaptic and neural excitability genes, it is unlikely that miR-27b's effect on neuronal activity is dominated by any one gene (other than transcriptional repressors). Nevertheless, miR-27b turns on or off several key genes implicated in synaptic transmission. Perhaps the most obvious candidate is the calcium sensor synaptotagmin-1 (Syt1), an abundant integral protein targeted to SVs that controls both evoked and spontaneous neurotransmitter release [45]. miR-27b elevates Syt1 expression by more than 2-fold in both the microarray and nCounter datasets. Syt1 is ranked third among down-regulated presynaptic genes and is within the top $0.2 \%$ most down-regulated genes (genome-wide) in miR-27b KD neurons. It is also substantially repressed by Bmil. Because Syt1 is required for evoked, synchronous neurotransmitter release [46, 47], it is possible that its down-regulation in miR-27b KD neurons contributes to reduced network activity. It is improbable, however, that Syt1 plays a major role in miR-27b-dependent regulation of mEPSC frequency, as Syt1 ablation results, instead, in an increase in mEPSC frequency [48], attributed to a clamp-like activity of Syt1 in resting cells. Several other presynaptic genes, all up-regulated more than two-fold by miR-27b, are likely to contribute to this synaptic deficit. These include Lin7a (Veli/MALS), an active zone scaffold [49], Slc17a7 (VGLUT1), recently implicated in coordination of SV cargo endocytosis [50], Rims2 (RIM2) and Syn2 (Synapsin II), which regulate vesicle docking at the active zone [51], and Snapin, a $15 \mathrm{kDa}$ protein that accelerates SV recycling [52]. Collectively, both the genomics and neurophysiology data point to a global and positive influence of miR-27b on presynaptic function. It should be noted, however, that miR-27b also silences a handful (44) of presynaptic genes, including positive regulators of neurotransmitter release, such as Liprin- $\alpha 4$ (Ppfia4) and Syntaxin-1A (Stx1A). Thus, the impact of miR-27b on neurotransmission is complex and likely involves bidirectional regulation of multiple synaptic components.

\section{Conclusion}

We describe in this paper a post-transcriptional circuitry that enables a single miRNA to exert considerable influence on the expression of functionally related genes. This network is based on miRNA-directed silencing of transcriptional repressors with overlapping, ontology-related targets, and departs from current models of miRNA-gene interactions, which ascribe to miRNAs a widespread, but modest effect on gene expression [53]. This circuitry may be well suited to drive irreversible cellular responses, such as cell differentiation. 


\section{Methods}

Mouse strains, DNA constructs and lentiviruses

$\operatorname{Dicer}^{f l / f l}$ mice were first described in [54] and were obtained from Jackson Laboratory (Dicer $1^{\text {tm1Bdh }}$, \#006366). The primers used for genotyping are described in [54]. miRZip-Scr (Cat: MZIP000-PA-1) and miRZip-27b (Cat: MZIP27b-PA-1) were purchased from Systems Biosciences (SBI). The anti-miR-27b shRNA targets both mouse and rat miR-27b-3p. The small hairpin sequence targeting miR-27b and its corresponding control (Scr) were subcloned in a modified pFUGW lentiviral vector containing an additional U6 promotor to drive expression of shRNAs (pFU6UGW). In some experiments, we used a red version of these constructs by replacing GFP with mCherry. The Cre-IRES-GFP construct was a gift from Dr. S. Je (Duke-NUS Medical School, Singapore). Cre-IRESGFP was subcloned into a modified pFUGW lentiviral vector that lacks GFP. Mouse Bmi1-IRES-GFP (\#21577) and Bmi1-shRNA (\#21576) were obtained from Addgene. Both constructs were made in a pFUGW backbone vector. Lentiviral particles based on the pFUGW vector were generated as previously described [24]. A multiplicity of infection (MOI) between two and five was used for all viral transduction experiments. Lentiviral particles expressing shRNAs against mouse Sox11 (Cat: V3SR760110EG498945) and Zfp90 (Cat: V3SR7601-10EG84046) were purchased from Dharmacon. Luciferase sensors were generated by PCR amplification and cloning of 3'UTRs into psiCHECK2 [55]. All oligos used in this study are listed in Additional file 10: Table S7 and all DNA constructs were sequenced before use. Antibodies against VGLUT1 (MAB5502, Chemicon), VGAT (131003, SySy) and Bassoon (ab82958, Abcam) were used for immunofluorescence studies.

\section{Primary mouse and rat neuron cultures}

Mouse cortical neurons were isolated from embryonic E16 Dicer1 ${ }^{f l f l}$ mouse embryos and dissociated with papain as described previously [56]. 500,000 neurons were seeded on 12-well poly-L-lysine-coated tissue culture dishes. Neurons were transduced with lentiviruses $3 \mathrm{~h}$ after plating and cultured in Neurobasal media with B27 supplement. For nCounter analysis, cell lysates were prepared from DIV14 neurons using $150 \mu \mathrm{l}$ of RLT buffer (Qiagen) per well. RNA was also extracted from the same three neuronal preparations for qPCR analysis using Sepasol RNA I Super G. For microarray analysis, RNA was isolated from DIV14 neurons using the RNeasy Mini kit (Qiagen) and RNA quality was analysed on a Bioanalyzer 2100 (Agilent Technologies). Rat cortical and hippocampal neurons were isolated from E18 rat embryos as previously described [24]. Lentiviruses were added $3 \mathrm{~h}$ or a day after plating. For electrophysiology recordings, 100,000 rat hippocampal neurons were seeded on $18 \mathrm{~mm}$ poly-L- lysine-coated glass coverslips and grown for 15-18 days before patching. For MEA recordings, 100,000 rat cortical neurons were seeded in MEA culture dishes and grown for 15 days before network activity was measured. For qPCR, RNA was extracted from 500,000 to 750,000 cortical neurons (DIV14) grown on poly-L-Lysine-coated 12-well tissue culture dish. For luciferase assays, 1 million cortical neurons were electroporated with luciferase sensors and seeded in poly-L-lysine-coated 24-well tissue culture plate and cell lysates were prepared after a week in culture.

\section{nCounter profiling of the presynaptic transcriptome}

We compiled a list of 195 "presynaptic" genes from the following papers and reviews [21, 57-60]. This list consists of genes whose products have been identified through biochemical/proteomics approaches in pre-synapses (or sub-structures of presynaptic terminals), and/or have been functionally implicated in presynaptic functions. We added to that list known miR-27b targets: Runx1 [61], Rxra [62] and Bmi1 [27] and 6 reported Dicer1 targets (Appbp2, Tgfbr3, Ccny, Neo1, Exd2, Dnmt3a) [63], four of which are also predicted miR-27b targets (Appbp2, Tgfbr3, Ccny, and Neo1). Seven genes were selected for signal normalization-4 ribosomal subunits (Rpl24, Rpl30, Rps16, Rps20), Gapdh, Cript and Cnga2. These normalization genes contain no predicted miRNA binding sites and have been defined by others as housekeeping genes in the CNS [64, 65]. A total of 212 nCounter probes were designed against these genes and synthesized by Nanostring Technologies (Seattle, WA). Accession numbers of these genes and probe sequences are listed in Additional file 1: Table S1. Hybridization and single-molecule detection of these probes were conducted on the nCounter multiplex platform at the Genome Institute of Singapore or in the S. Albani lab (SingHealth Translational Immunology and Inflammation Centre, Singapore). Probe counts were analysed with the nSolver software (Nanostring Technologies). We used the geometric mean of six normalization genes (Cnga2 was discarded because of its low counts) as normalization factor. Triplicates were run, each corresponding to an independent neuron preparation with both miR-27b CT and KD conditions. We used log2transformed fold-change (FC) to display differential gene expression: $\log 2 \mathrm{FC}=\log 2(\mathrm{KD})-\log 2(\mathrm{CT}) ; \mathrm{FC}=2^{\wedge} \log 2 \mathrm{FC}$. For combinatorial intersection analysis of nCounter datasets, we used the open source CRAN R package UpSet [66].

\section{Microarray profiling and Gene Set Enrichment Analysis (GSEA)}

Microarray analysis was performed by the Duke-NUS Genome Biology Facility on an Illumina platform (San 
Diego, CA). Biotin-labeled cRNA was prepared using the TotalPrep RNA amplification Kit (Ambion) from $500 \mathrm{ng}$ total RNA. 1.5 ug of biotin-labeled cRNA was hybridized to MouseWG-6 v2.0 expression beadchips, and chips were washed and stained according the manufacturer's instructions. Beadchips were scanned using the Illumina BeadArray reader. Expression data were analysed using the R-Bioconductor open source software (limma R package) and are derived from triplicate experiments, each corresponding to one neuronal preparation with both miR-27b CT and KD conditions. Genes with foldchange $>1.5$ and a false discovery rate $<0.05$ were considered as differentially-regulated. Hierarchical clustering, dendrograms and heat maps were performed and displayed using the clustergram function in Matlab. For GSEA analysis of microarray data, we used the GSEA software available on the GSEA-Broad Institute website. We ran our expression dataset against a library of 1330 curated gene sets for canonical pathways (c2.cp.v5.1. symbols.gmt; MSigDB v 5.1). Qualitatively similar results were obtained with a gene ontology (GO) gene set library (c2.bp.v5.1.symbols.gmt). The statistical significance (nominal $\mathrm{p}$ value) of the enrichment score (ES) was estimated by running 1000 gene set permutations. The ES was normalized (NES) to account for the size of the gene set. To adjust for multiple testing across the 1330 gene sets, we computed the false discovery rate (FDR) and controlled the FDR at $25 \%$. Gene networks were visualized using the open source software platform Cytoscape 3.3.

\section{RT-qPCR and luciferase assays}

For quantification of miRNA levels in mouse neurons, we used Taqman RT and qPCR primers (Applied Biosystems) specific for Y1 scRNA (control), miR-27b-3p, and miR-181a, as previously described [24] Y1 was used for normalization. For all other RT-qPCR experiments, RNA was extracted from DIV14 mouse or rat neurons using the Qiagen RNeasy Mini kit. qPCR primers used for mouse Trib3, Mex3a, Sox11, Zfp90, Bmi1, and rat Bmi1 are described in Additional file 10: Table S7. Reverse transcription was done using the iScript cDNA synthesis kit (BioRad). qPCR reactions were run in triplicates with iQ SYBR Green supermix (BioRad) and KiCqStart SYBR Green primers (Sigma). Reactions were performed on the Bio-Rad CFX-96 machine (BioRad) and actin was used for normalization. Luciferase assays were conducted using the Dual-Luciferase Reporter kit (Promega) as described previously [24]. Luciferase sensors tagged with the 3'UTR of rat Trib3, St8sia2, Mex3a, Sox11, Sh3bp2, Zfp90 and Bmil were introduced in rat cortical neurons by nucleofection (Amaxa, Lonza). After a week in culture, firefly and renilla luminescences were sequentially measured and Renilla counts (under 3'UTR control) were normalized to firefly counts.

Whole-cell recordings and multi-electrodes arrays (MEAs) Whole-cell patch-clamp recordings were performed in DIV15-18 hippocampal neurons, using a multi-Clamp 700B amplifier driven by the pClamp software (Axon Instruments, Sunnyvale, CA), as previously described [67]. Cells were held at $-70 \mathrm{mV}$. Patch pipettes (3-5 $\mathrm{M} \Omega$ resistance) were filled with an internal solution containing (in $\mathrm{mM}$ ) $120 \mathrm{~K}$-Gluconate, $9 \mathrm{KCl}, 10 \mathrm{KOH}, 3.48 \mathrm{MgCl}_{2}$, $4 \mathrm{NaCl}, 10$ HEPES, $4 \mathrm{Na}_{2} \mathrm{ATP}, 0.4 \mathrm{Na}_{3} \mathrm{GTP}, 19.5$ Sucrose, 5 EGTA. Neurons were bathed in external solution containing (in $\mathrm{mM}$ ) $110 \mathrm{NaCl}, 5 \mathrm{KCl}, 2 \mathrm{CaCl}_{2}, 0.8 \mathrm{MgCl}_{2}, 10$ HEPES pH 7.4 and 10 D-glucose and supplemented with $0.5 \mu \mathrm{M}$ TTX, to prevent action potential-evoked EPSCs, and $10 \mu \mathrm{M}$ BMI to block GABAergic inhibitory postsynaptic potentials. Data were analyzed using pClamp. Only recording epochs in which series and input resistances varied by $<10 \%$ were included in the analysis. The identity of miR-27b CT and KD groups was concealed from the experimenter. For MEA recordings, rat cortical neurons were plated on culture dishes containing an array of 60 (8 x 8) electrodes (Multi-Channel Systems). Extracellular recordings were performed on an air-suspended table in a Faraday cage. Signals were amplified using the MEA1060 hardware system (Multi-Channel Systems), and recorded in real-time for $5 \mathrm{~min}$ using the MC_rack software (Multi-Channel Systems). High pass $(300 \mathrm{~Hz})$ and low pass $(3 \mathrm{kHz})$ filters were applied to the data. Spikes were detected using a voltage threshold rule (standard deviation $>6$ ). Data were transferred to Matlab to determine the frequency of population bursts using a customdesigned script.

\section{Immunocytochemistry and image analysis of SV puncta}

Hippocampal neurons (DIV15) grown on glass coverslips were fixed in $4 \%$ PFA, $4 \%$ sucrose, permeabilized with $100 \mathrm{ug} / \mathrm{ml}$ digitonin for $10 \mathrm{~min}$ and blocked with $5 \%$ goat serum for $1 \mathrm{~h}$. Fixed cells were co-incubated with primary Abs against VGLUT1 (mouse Ab) and VGAT (rabbit Ab) and secondary Abs conjugated to Alexa Fluor 568 (antimouse IgG) or 633 (anti-rabbit IgG). Neurons were then imaged for GFP, VGLUT1 and VGAT using a laser scanning confocal microscope (LSM710, Zeiss). Puncta density and intensity was quantified using Matlab-based scripts described previously $[24,68]$.

\section{Statistical analysis}

Statistical tests used for the genomics data (nCounter, microarray and GSEA) were described in previous sections and in figure legends. Average data are represented as means \pm SD unless stated otherwise. Statistical significance was determined using two-tailed unpaired t-tests 
on data sets consisting of two groups. One-way ANOVA was used when simultaneously comparing three or more data sets. In this case, $\mathrm{p}$ values were derived from a post-hoc Bonferroni test.

\section{Additional files}

Additional file 1: Table S1. List of nCounter probes used in this study. (XLSX $26 \mathrm{~kb}$ )

Additional file 2: Table S2. Differentially-expressed presynaptic genes in miR-27b KD (A) and Dicer1 CKO (B) neurons. (nCounter dataset). (XLSX 73 kb) Additional file 3: Table S3. Up (A) and Down (B) regulated genes in miR-27b KD neurons (microarray dataset). (XLSX $380 \mathrm{~kb}$ )

Additional file 4: Table S4. Comparative analysis of the presynaptic transcriptome in miR-27b KD neurons by $\mathrm{nCounter}$ or microarray profiling. (XLSX $11 \mathrm{~kb}$ )

Additional file 5: Figure S1. Microarray analysis of the presynaptic transcriptome in miR-27 KD $(n=3)$ and $C T(n=3)$ neurons. Shown are differentially-expressed genes (FDR $<0.05$ ). (TIF $235 \mathrm{~kb}$ )

Additional file 6: Table S5. List of down-regulated gene sets in miR-27 KD neurons. (XLSX $21 \mathrm{~kb}$ )

Additional file 7: Table S6. List of up-regulated gene sets in miR-27 KD neurons. (XLSX $18 \mathrm{~kb}$ )

Additional file 8: Figure S2. Gene networks visualized using Cytoscape 3.3 and generated from both up- (red) and down- (blue) regulated gene sets in miR-27 KD neurons. (TIF $1178 \mathrm{~kb}$ )

Additional file 9: Figure S3. Bassoon immunostaining in miR-27 KD and CT hippocampal neurons. (TIF $765 \mathrm{~kb}$ )

Additional file 10: Table S7. List of DNA primers used in this study. (XLSX $11 \mathrm{~kb})$

\section{Acknowledgments}

We thank Dr. Sujoy Ghosh for critical reading of the manuscript, Dr. Mathijs Voorhoeve for his input in miRNA biology and Paul Rasmussen (NanoString Technologies) for invaluable advice on $\mathrm{nCounter}$ experiments. We are grateful to the Duke-NUS genomic facility for running the microarray and Thomas Thurnherr for help with $\mathrm{R}$ analysis of microarray data. We would also like to thank the Christopher Wong and Salvatore Albani Labs for giving us access to their nCounter platform, and we are indebted to Xiaoyu Zhang, How Wing Leung and Fang-Chin Yeh for help with the MEA experiments.

\section{Funding}

This work was supported by grants from the Ministry of Education Academic Research Fund (LKYPDF-2011-02) and the National Medical Research Council (NMRC/BNIG1/2022/2014) to VYP, by an EDG NMRC grant (EDG10nov064) to MF and by the Duke-NUS Signature Research Program funded by the Agency for Science, Technology and Research (A*STAR) and the Ministry of Health $(\mathrm{MOH})$, Singapore to MF.

\section{Availability of data and materia}

The microarray dataset supporting the conclusions of this article is publicly available at Gene Expression Omnibus (GEO-NCBI, accession number GSE73275). The other datasets supporting the conclusions of this article are in included within the article and its additional files.

\section{Authors' contributions}

VYP and MG performed all biochemical and MEA experiments. FJ carried out whole-cell recordings. AVD gave us access to his MEA setup and provided expertise in MEA recordings. VYP MG and MF analysed data. MF wrote the manuscript. All authors read and approved the final manuscript.

\section{Competing interests}

The authors declare that they have no competing interests.
Consent for publication

Not applicable.

Ethics approval and consent to participate

All animal procedures were approved by the SingHealth Institutional Animal Care and Use Committee (IACUC) of Singapore (Fivaz laboratory protocol \# 2011/SHS/618).

Received: 14 May 2016 Accepted: 28 September 2016

Published online: 04 October 2016

\section{References}

1. Lewis BP, Burge CB, Bartel DP. Conserved seed pairing, often flanked by adenosines, indicates that thousands of human genes are microRNA targets. Cell. 2005;120:15-20.

2. Guo H, Ingolia NT, Weissman JS, Bartel DP. Mammalian microRNAs predominantly act to decrease target mRNA levels. Nature. 2010;466:835-40.

3. Bazzini AA, Lee MT, Giraldez AJ. Ribosome profiling shows that miR-430 reduces translation before causing mRNA decay in zebrafish. Science. 2012;336:233-7.

4. Djuranovic S, Nahvi A, Green R. miRNA-mediated gene silencing by translational repression followed by mRNA deadenylation and decay. Science. 2012:336:237-40.

5. Chi SW, Zang JB, Mele A, Darnell RB. Argonaute HITS-CLIP decodes microRNA-mRNA interaction maps. Nature. 2009:460:479-86.

6. Boudreau RL, Jiang P, Gilmore BL, Spengler RM, Tirabassi R, Nelson JA, Ross CA, Xing Y, Davidson BL. Transcriptome-wide discovery of microRNA binding sites in human brain. Neuron. 2014;81:294-305.

7. Fineberg SK, Kosik KS, Davidson BL. MicroRNAs potentiate neural development. Neuron. 2009;64:303-9.

8. Wakabayashi T, Hidaka R, Fujimaki S, Asashima M, Kuwabara T. MicroRNAs and epigenetics in adult neurogenesis. Adv Genet. 2014;86:27-44.

9. Smrt RD, Szulwach KE, Pfeiffer RL, Li X, Guo W, Pathania M, Teng ZQ, Luo Y, Peng J, Bordey A, et al. MicroRNA miR-137 regulates neuronal maturation by targeting ubiquitin ligase mind bomb-1. Stem Cells. 2010;28:1060-70.

10. Tan $C L$, Plotkin JL, Veno MT, von Schimmelmann M, Feinberg P, Mann S, Handler A, Kjems J, Surmeier DJ, O'Carroll D, et al. MicroRNA-128 governs neuronal excitability and motor behavior in mice. Science. 2013;342:1254-8.

11. Sosanya NM, Huang PP, Cacheaux LP, Chen CJ, Nguyen K, Perrone-Bizzozero NI, Raab-Graham KF. Degradation of high affinity HuD targets releases Kv1.1 mRNA from miR-129 repression by mTORC1. J Cell Biol. 2013;202:53-69.

12. Siegel G, Obernosterer G, Fiore R, Oehmen M, Bicker S, Christensen M, Khudayberdiev S, Leuschner PF, Busch CJ, Kane C, et al. A functional screen implicates microRNA-138-dependent regulation of the depalmitoylation enzyme APT1 in dendritic spine morphogenesis. Nat Cell Biol. 2009;11:705-16.

13. Lippi G, Steinert JR, Marczylo EL, D'Oro S, Fiore R, Forsythe ID, Schratt G, Zoli M, Nicotera P, Young KW. Targeting of the Arpc3 actin nucleation factor by miR-29a/b regulates dendritic spine morphology. J Cell Biol. 2011;194:889-904

14. Hu Z, Yu D, Gu QH, Yang Y, Tu K, Zhu J, Li Z. miR-191 and miR-135 are required for long-lasting spine remodelling associated with synaptic long-term depression. Nat Commun. 2014;5:3263.

15. Edbauer D, Neilson JR, Foster KA, Wang CF, Seeburg DP, Batterton MN, Tada T, Dolan BM, Sharp PA, Sheng M. Regulation of synaptic structure and function by FMRP-associated microRNAs miR-125b and miR-132. Neuron. 2010;65:373-84.

16. Saba R, Storchel PH, Aksoy-Aksel A, Kepura F, Lippi G, Plant TD, Schratt GM. Dopamine-regulated microRNA MiR-181a controls GluA2 surface expression in hippocampal neurons. Mol Cell Biol. 2012;32:619-32.

17. Muddashetty RS, Nalavadi VC, Gross C, Yao X, Xing L, Laur O, Warren ST, Bassell GJ. Reversible inhibition of PSD-95 mRNA translation by miR-125a, FMRP phosphorylation, and mGluR signaling. Mol Cell. 2011;42:673-88.

18. Scott HL, Tamagnini F, Narduzzo KE, Howarth JL, Lee YB, Wong LF, Brown MW, Warburton EC, Bashir Zl, Uney JB. MicroRNA-132 regulates recognition memory and synaptic plasticity in the perirhinal cortex. Eur J Neurosci. 2012;36:2941-8.

19. Remenyi J, van den Bosch MW, Palygin O, Mistry RB, McKenzie C, Macdonald A, Hutvagner G, Arthur JS, Frenguelli BG, Pankratov Y. miR132/212 knockout mice reveal roles for these miRNAs in regulating cortical synaptic transmission and plasticity. PLoS One. 2013;8:e62509. 
20. Liu R, Hannenhalli S, Bucan M. Motifs and cis-regulatory modules mediating the expression of genes co-expressed in presynaptic neurons. Genome Biol. 2009; 10:R72.

21. Paschou M, Paraskevopoulou MD, Vlachos IS, Koukouraki P, Hatzigeorgiou AG, Doxakis E. miRNA regulons associated with synaptic function. PLoS One. 2012;7:e46189.

22. Verma P, Augustine GJ, Ammar MR, Tashiro A, Cohen SM. A neuroprotective role for microRNA miR-1000 mediated by limiting glutamate excitotoxicity. Nat Neurosci. 2015;18:379-85

23. Tsurudome K, Tsang K, Liao EH, Ball R, Penney J, Yang JS, Elazzouzi F, He T, Chishti A, Lnenicka G, et al. The Drosophila miR-310 cluster negatively regulates synaptic strength at the neuromuscular junction. Neuron. 2010;68:879-93.

24. Poon W, Goh C, Voorhoeve PM, Fivaz M. High-content imaging of presynaptic assembly. Front Cell Neurosci. 2014;8:66.

25. Moreau MP, Bruse SE, David-Rus R, Buyske S, Brzustowicz LM. Altered microRNA expression profiles in postmortem brain samples from individuals with schizophrenia and bipolar disorder. Biol Psychiatry. 2011;69:188-93.

26. Geiss GK, Bumgarner RE, Birditt B, Dahl T, Dowidar N, Dunaway DL, Fell HP Ferree S, George RD, Grogan T, et al. Direct multiplexed measurement of gene expression with color-coded probe pairs. Nat Biotechnol. 2008;26:317-25.

27. Guerau-de-Arellano M, Smith KM, Godlewski J, Liu Y, Winger R, Lawler SE, Whitacre CC, Racke MK, Lovett-Racke AE. Micro-RNA dysregulation in multiple sclerosis favours pro-inflammatory T-cell-mediated autoimmunity. Brain. 2011;134:3578-89.

28. Bhattacharya R, Mustafi SB, Street M, Dey A, Dwivedi SK. Bmi-1: At the crossroads of physiological and pathological biology. Genes Dis. 2015;2:225-39.

29. Fasano CA, Phoenix TN, Kokovay E, Lowry N, Elkabetz Y, Dimos JT, Lemischka IR, Studer L, Temple S. Bmi-1 cooperates with Foxg1 to maintain neural stem cell self-renewal in the forebrain. Genes Dev. 2009:23:561-74.

30. Bergsland M, Ramskold D, Zaouter C, Klum S, Sandberg R, Muhr J. Sequentially acting Sox transcription factors in neural lineage development. Genes Dev. 2011;25:2453-64.

31. Mu L, Berti L, Masserdotti G, Covic M, Michaelidis TM, Doberauer K, Merz K, Rehfeld F, Haslinger A, Wegner M, et al. SoxC transcription factors are required for neuronal differentiation in adult hippocampal neurogenesis. J Neurosci. 2012;32:3067-80.

32. Chen C, Lee GA, Pourmorady A, Sock E, Donoghue MJ. Orchestration of neuronal differentiation and progenitor pool expansion in the developing cortex by SoxC genes. J Neurosci. 2015;35:10629-42.

33. Tsurusaki Y, Koshimizu E, Ohashi H, Phadke S, Kou I, Shiina M, Suzuki T, Okamoto N, Imamura S, Yamashita M, et al. De novo SOX11 mutations cause Coffin-Siris syndrome. Nat Commun. 2014;5:4011.

34. Hata L, Murakami M, Kuwahara K, Nakagawa Y, Kinoshita H, Usami S, Yasuno S, Fujiwara M, Kuwabara Y, Minami T, et al. Zinc-finger protein 90 negatively regulates neuron-restrictive silencer factor-mediated transcriptional repression of fetal cardiac genes. J Mol Cell Cardiol. 2011:50:972-81.

35. Palka Bayard de Volo C, Alfonsi M, Gatta V, Novelli A, Bernardini L, Fantasia D, Antonucci I, Angelucci D, Zori R, Stuppia L, et al. 16q22.1 microdeletion detected by array-CGH in a family with mental retardation and lobular breast cancer. Gene. 2012:498:328-31.

36. Subramanian A, Tamayo P, Mootha VK, Mukherjee S, Ebert BL, Gillette MA, Paulovich A, Pomeroy SL, Golub TR, Lander ES, Mesirov JP. Gene set enrichment analysis: a knowledge-based approach for interpreting genomewide expression profiles. Proc Natl Acad Sci U S A. 2005;102:15545-50.

37. Mootha VK, Lindgren CM, Eriksson KF, Subramanian A, Sihag S, Lehar J, Puigserver P, Carlsson E, Ridderstrale M, Laurila E, et al. PGC-1alpharesponsive genes involved in oxidative phosphorylation are coordinately downregulated in human diabetes. Nat Genet. 2003;34:267-73.

38. Shannon P, Markiel A, Ozier O, Baliga NS, Wang JT, Ramage D, Amin N, Schwikowski B, Ideker T. Cytoscape: a software environment for integrated models of biomolecular interaction networks. Genome Res. 2003;13:2498-504

39. Siciliano V, Garzilli I, Fracassi C, Criscuolo S, Ventre S, di Bernardo D. MiRNAs confer phenotypic robustness to gene networks by suppressing biological noise. Nat Commun. 2013:4:2364.

40. Liu B, Li J, Tsykin A, Liu L, Gaur AB, Goodall GJ. Exploring complex miRNAmRNA interactions with Bayesian networks by splitting-averaging strategy. BMC Bioinformatics. 2009;10:408.
41. Simon JA, Kingston RE. Occupying chromatin: Polycomb mechanisms for getting to genomic targets, stopping transcriptional traffic, and staying put. Mol Cell. 2013;49:808-24.

42. Molofsky AV, Pardal R, Iwashita T, Park IK, Clarke MF, Morrison SJ. Bmi-1 dependence distinguishes neural stem cell self-renewal from progenitor proliferation. Nature. 2003:425:962-7.

43. Mochida GH, Ganesh VS, de Michelena MI, Dias H, Atabay KD, Kathrein KL, Huang HT, Hill RS, Felie JM, Rakiec D, et al. CHMP1A encodes an essential regulator of BMI1-INK4A in cerebellar development. Nat Genet. 2012;44:1260-4.

44. Liguz-Lecznar M, Skangiel-Kramska J. Vesicular glutamate transporters (VGLUTs): the three musketeers of glutamatergic system. Acta Neurobiol Exp (Wars). 2007:67:207-18

45. Chapman ER. How does synaptotagmin trigger neurotransmitter release? Annu Rev Biochem. 2008:77:615-41.

46. Nishiki T, Augustine GJ. Dual roles of the C2B domain of synaptotagmin I in synchronizing Ca2 + -dependent neurotransmitter release. J Neurosci. 2004;24:8542-50.

47. Nishiki T, Augustine GJ. Synaptotagmin I synchronizes transmitter release in mouse hippocampal neurons. J Neurosci. 2004;24:6127-32.

48. Pang ZP, Sun J, Rizo J, Maximov A, Sudhof TC. Genetic analysis of synaptotagmin 2 in spontaneous and Ca2 + -triggered neurotransmitter release. EMBO J. 2006;25:2039-50.

49. Olsen O, Moore KA, Fukata M, Kazuta T, Trinidad JC, Kauer FW, Streuli M, Misawa H, Burlingame AL, Nicoll RA, Bredt DS. Neurotransmitter release regulated by a MALS-liprin-alpha presynaptic complex. J Cell Biol. 2005;170:1127-34.

50. Pan PY, Marrs J, Ryan TA. Vesicular glutamate transporter 1 orchestrates recruitment of other synaptic vesicle cargo proteins during synaptic vesicle recycling. J Biol Chem. 2015;290:22593-601.

51. Han Y, Kaeser PS, Sudhof TC, Schneggenburger R. RIM determines Ca(2) + channel density and vesicle docking at the presynaptic active zone. Neuron. 2011;69:304-16

52. Di Giovanni J, Sheng ZH. Regulation of synaptic activity by snapin-mediated endolysosomal transport and sorting. EMBO J. 2015:34:2059-77.

53. Iwama $\mathrm{H}$. Coordinated networks of microRNAs and transcription factors with evolutionary perspectives. Adv Exp Med Biol. 2013;774:169-87.

54. Harfe BD, McManus MT, Mansfield JH, Hornstein E, Tabin CJ. The RNaselll enzyme Dicer is required for morphogenesis but not patterning of the vertebrate limb. Proc Natl Acad Sci U S A. 2005;102:10898-903.

55. Beillard E, Ong SC, Giannakakis A, Guccione E, Vardy LA, Voorhoeve PM. miR-Sens-a retroviral dual-luciferase reporter to detect microRNA activity in primary cells. RNA. 2012;18:1091-100

56. Garcia-Alvarez G, Shetty MS, Lu B, Yap KA, Oh-Hora M, Sajikumar S, Bichler Z, Fivaz M. Impaired spatial memory and enhanced long-term potentiation in mice with forebrain-specific ablation of the Stim genes. Front Behav Neurosci. 2015;9:180

57. Boyken J, Gronborg M, Riedel D, Urlaub H, Jahn R, Chua JJ. Molecular profiling of synaptic vesicle docking sites reveals novel proteins but few differences between glutamatergic and GABAergic synapses. Neuron. 2013;78:285-97

58. Clarke GL, Chen J, Nishimune $H$. Presynaptic active zone density during development and synaptic plasticity. Front Mol Neurosci. 2012;5:12.

59. Sudhof TC. The presynaptic active zone. Neuron. 2012;75:11-25.

60. Waites CL, Garner CC. Presynaptic function in health and disease. Trends Neurosci. 2011:34:326-37.

61. Feng J, Iwama A, Satake M, Kohu K. MicroRNA-27 enhances differentiation of myeloblasts into granulocytes by post-transcriptionally downregulating Runx1. Br J Haematol. 2009;145:412-23.

62. Ji J, Zhang J, Huang G, Qian J, Wang X, Mei S. Over-expressed microRNA$27 \mathrm{a}$ and $27 \mathrm{~b}$ influence fat accumulation and cell proliferation during rat hepatic stellate cell activation. FEBS Lett. 2009;583:759-66.

63. Dorval V, Smith PY, Delay C, Calvo E, Planel E, Zommer N, Buee L, Hebert SS Gene network and pathway analysis of mice with conditional ablation of Dicer in post-mitotic neurons. PLoS One. 2012;7:e44060.

64. Cajigas IJ, Tushev G, Will TJ, tom Dieck S, Fuerst N, Schuman EM. The local transcriptome in the synaptic neuropil revealed by deep sequencing and high-resolution imaging. Neuron. 2012;74:453-66.

65. Khan $M$, Vaes $E$, Mombaerts $P$. Regulation of the probability of mouse odorant receptor gene choice. Cell. 2011;147:907-21. 
66. Lex A, Gehlenborg N, Strobelt H, Vuillemot R, Pfister H. UpSet: Visualization of Intersecting Sets. IEEE Trans Vis Comput Graph. 2014;20:1983-92.

67. Garcia-Alvarez G, Lu B, Yap KA, Wong LC, Thevathasan JV, Lim L, Ji F, Tan KW, Mancuso JJ, Tang W, et al. STIM2 regulates PKA-dependent phosphorylation and trafficking of AMPARs. Mol Biol Cell. 2015;26(6):1141-59.

68. Tang W, Thevathasan JV, Lin Q, Lim KB, Kuroda K, Kaibuchi K, Bilger M, Soong TW, Fivaz M. Stimulation of synaptic vesicle exocytosis by the mental disease gene DISC1 is mediated by N-type voltage-gated calcium channels. Front Synaptic Neurosci. 2016;8:15.

Submit your next manuscript to BioMed Central and we will help you at every step:

- We accept pre-submission inquiries

- Our selector tool helps you to find the most relevant journal

- We provide round the clock customer support

- Convenient online submission

- Thorough peer review

- Inclusion in PubMed and all major indexing services

- Maximum visibility for your research

Submit your manuscript at www.biomedcentral.com/submit 